\title{
Synonymous Dinucleotide Usage: A Codon-Aware Metric for Quantifying Dinucleotide Representation in Viruses
}

\author{
Spyros Lytras * and Joseph Hughes ${ }^{(1)}$ \\ MRC-University of Glasgow Centre for Virus Research, Glasgow G61 1QH, UK \\ * Correspondence: s.lytras.1@research.gla.ac.uk
}

Received: 28 February 2020; Accepted: 17 April 2020; Published: 20 April 2020

check for updates

\begin{abstract}
Distinct patterns of dinucleotide representation, such as CpG and UpA suppression, are characteristic of certain viral genomes. Recent research has uncovered vertebrate immune mechanisms that select against specific dinucleotides in targeted viruses. This evidence highlights the importance of systematically examining the dinucleotide composition of viral genomes. We have developed a novel metric, called synonymous dinucleotide usage (SDU), for quantifying dinucleotide representation in coding sequences. Our method compares the abundance of a given dinucleotide to the null hypothesis of equal synonymous codon usage in the sequence. We present a Python3 package, DinuQ, for calculating SDU and other relevant metrics. We have applied this method on two sets of invertebrate- and vertebrate-specific flaviviruses and rhabdoviruses. The SDU shows that the vertebrate viruses exhibit consistently greater under-representation of $\mathrm{CpG}$ dinucleotides in all three codon positions in both datasets. In comparison to existing metrics for dinucleotide quantification, the SDU allows for a statistical interpretation of its values by comparing it to a null expectation based on the codon table. Here we apply the method to viruses, but coding sequences of other living organisms can be analysed in the same way.
\end{abstract}

Keywords: dinucleotides; CpG suppression; Flaviviridae; Rhabdoviridae; synonymous codon usage; bioinformatics; python package

\section{Introduction}

Certain dinucleotides, two nucleotides adjacent in a sequence, are known to be over- or under-represented in the genomes of living organisms, creating distinct compositional patterns [1]. Organisms with methylated genomes such as vertebrates and plants have low levels of CpG dinucleotides. This is not the case for methylase-absent organisms like invertebrates, bacteria and fungi $[2,3]$. The cause of CpG suppression lies in the DNA methylation mechanisms of vertebrates and plants, where cytosine is frequently converted to thymine by DNA methyltransferases [4,5]. UpA deprivation is consistently present in most living organisms, including prokaryotes. This bias is suspected to be due to UpA-rich mRNA being unstable and more prone to degradation by cytoplasmic RNAses [6,7].

Similar patterns of dinucleotide composition have been observed in RNA and DNA viruses, and appear to have a functional role in the infection and propagation of the viruses [8]. Studies have shown that experimentally increasing UpA and CpG levels in RNA viruses leads to a decrease in replication and subsequent viral attenuation [9-11]. Influenza CpG-/UpA-rich sequences have also been shown to have reduced replication in vivo, causing a more powerful immune response, and in the case of $\mathrm{CpG}$ increase, showing reduced clinical severity [12]. A similar decrease in replication and virulence has been observed in CpG-/UpA-increased yellow fever virus [13]. 
Recently, a host immune mechanism of vertebrates which acts on viral genomes on the dinucleotide level, has been uncovered. The zinc-finger antiviral protein (ZAP) appears to be integral to this system by targeting CpG-rich infecting viral RNA [14]. This host-driven force selects against viruses rich in CpG dinucleotides and drives the observed under-representation of CpG in HIV-1 [14], the model RNA virus Echovirus 7 [15], and even DNA viruses [16]. Additionally, UpA is mildly under-represented in most viral genomes [17]. Even though our understanding of this dinucleotide's depletion is limited, there is some evidence suggesting that a similar antiviral mechanism might also be responsible for this pattern $[15,18]$.

The genetic code is degenerate, meaning that some codons encode the same amino acid. These codons are called synonymous, because changes between them do not alter the resulting peptide sequence. The frequency in which synonymous codons are found in a coding sequence can be biased, as a result of various mutational or selective pressures acting on the genome [19]. Metrics for measuring codon usage bias exist, such as the relative synonymous codon usage (RSCU), which quantify the bias in synonymous codon frequencies [20]. However, these measures do not inform on the underlying dinucleotide bias in the sequence. If such forces are acting on the dinucleotide and not the peptide level in viral genomes, adaptation towards, for example, a less CpG-rich genome, would preferentially take place through synonymous changes in the sequence. This would lead to a genome with a reduced abundance of dinucleotide patterns recognized by host immunity, whilst still maintaining the protein sequence. Thus, the dinucleotide-acting compositional bias would produce a skew in the frequencies of synonymous codon usage in the genome.

Previous research has tried to disentangle dinucleotide patterns from codon and amino acid frequencies, mainly focusing on quantifying CpG patterns [21]. More complex mathematical analyses have also been implemented for understanding evolutionary change in viral genome nucleotide motifs [22]. The only method, however, that is being routinely used for quantifying dinucleotide representation is the relative dinucleotide abundance (RDA). This is simply the odds ratio between the frequency of a dinucleotide $X Y$ and the product of the frequencies of each single nucleotide $X$ and $Y$ across a whole sequence [23]. The evidence of a host mechanism selecting against dinucleotides in viruses highlights the importance of developing a method for systematically quantifying dinucleotide representation by comparing the observed patterns to a biologically relevant null hypothesis. We propose the synonymous dinucleotide usage (SDU), a metric that compares the occurrences of a given dinucleotide to the null hypothesis where there is equal usage of synonymous codons. This method provides a way of measuring the extent to which a host-driven force acting on the dinucleotide level of viral genomes has skewed the synonymous codon usage of the protein sequence and can be applied in all dinucleotide combinations and different sequence frame positions.

\section{Materials and Methods}

\subsection{Synonymous Dinucleotide Usage (SDU)}

A coding sequence can have three distinct dinucleotide frame positions. We define the dinucleotide frame position 1 as the first and second nucleotide position of a codon, dinucleotide frame position 2 as the second and third nucleotide position of a codon, and dinucleotide bridge position as the third nucleotide position of a codon and the first position of the downstream codon. Each one of these positions can take a set of different dinucleotides without changing the amino acid (positions 1 and 2) or amino acid pair (bridge position) in the protein sequence, we define these as a set of synonymous dinucleotides. For example, threonine has four synonymous codons ACU, ACC, ACA, ACG. In dinucleotide position 2 of a codon encoding for threonine, there are four synonymous dinucleotides, $\mathrm{CU}, \mathrm{CC}, \mathrm{CA}$, and CG. As such, the expected proportion of $\mathrm{CpU}$ occurring in position 2 coding for threonine under the null hypothesis of equal synonymous codon usage is: $e_{i}=0.25$. The SDU (Table 1 ) compares the observed proportion of a synonymous dinucleotide of interest $\left(\mathrm{o}_{\mathrm{i}}\right)$ to that expected under equal synonymous codon usage $\left(\mathrm{e}_{\mathrm{i}}\right)$ for a given dinucleotide frame position. The ratio between $\mathrm{o}_{\mathrm{i}}$ and 
$e_{i}$ is calculated for each different amino acid or amino acid pair (for the bridge position) and the SDU is defined as the weighted arithmetic mean of the ratios, weighted by the abundance of each amino acid in the sequence (Equation (1)).

$$
\operatorname{SDU}_{j, h}=\frac{\sum_{i=1}^{k} n_{i} \times \frac{o_{i, j, h}}{e_{i, j, h}}}{N}
$$

The set $j$ includes 16 possible dinucleotide combinations. With three frame positions $h$, the matrix of $\mathrm{SDU}_{\mathrm{j}, \mathrm{h}}$ has 48 possible combinations. Only 3 amino acids can be encoded by different position 1 dinucleotides (arginine, serine, and leucine), meaning that 11 out of 16 dinucleotides in frame position 1 are non-informative, leaving 37 informative combinations. The result of the SDU directly reflects the overall synonymous dinucleotide representation in each frame position of a given sequence:

- An SDU of 1 indicates that the representation of the dinucleotide of interest in the given frame position is equal to that expected under the null hypothesis of equal synonymous codon usage;

- an SDU of 0 indicates that the dinucleotide of interest is completely absent in the given frame position across the sequence;

- $\quad$ an SDU greater than 1 indicates that the dinucleotide of interest is over-represented in the given frame position, compared to the representation expected under the null hypothesis;

- an SDU between 0 and 1 indicates that the dinucleotide of interest is under-represented in the given frame position, compared to the representation expected under the null hypothesis.

Table 1. Notation used to define SDU and RSDU.

\begin{tabular}{|c|c|}
\hline Symbol & Description \\
\hline$i$ & Amino acid or amino acid pair \\
\hline$j$ & Dinucleotide \\
\hline$h$ & Dinucleotide frame position \\
\hline$n_{i}$ & $\begin{array}{c}\text { Number of occurrences of amino acid or amino acid pair } i \text { in the } \\
\text { sequence }\end{array}$ \\
\hline$k$ & Set of different amino acids or amino acid pairs present in the sequence \\
\hline$o_{i, j, h}$ & $\begin{array}{l}\text { Synonymous proportion of dinucleotide } j \text { in frame position } h \text { for amino } \\
\text { acid or amino acid pair } i \text { observed in the sequence }\end{array}$ \\
\hline$e_{i, j, h}$ & $\begin{array}{l}\text { Synonymous proportion of dinucleotide } j \text { in frame position } h \text { for amino } \\
\text { acid or amino acid pair } i \text { expected under equal synonymous codon usage }\end{array}$ \\
\hline$N$ & Total number of amino acids or amino acid pairs present in the sequence \\
\hline
\end{tabular}

\subsection{Relative Synonymous Dinucleotide Usage (RSDU)}

The number of amino acids or amino acid pairs that can be synonymously produced by a certain dinucleotide varies between dinucleotides and frame positions. This means that SDU measurements for different positions and dinucleotides can reach different maximum values. Under- (SDU $<1$ ) and over-representation (SDU >1) can still be consistently interpreted between positions and dinucleotides, since an SDU of 1 always reflects complete agreement with the null hypothesis. However, the magnitude of over-representation cannot be compared between different positions and dinucleotides. In order to make this comparison possible we extended the SDU to the relative synonymous dinucleotide usage (RSDU). This is essentially the calculated SDU value, normalised by the maximum SDU for this position and dinucleotide (Equation (2)). This extension to the metric does not have a consistent scale for comparison to the null hypothesis, and instead allows for comparing the extent of the difference from the null hypothesis between the different parameters.

$$
\operatorname{RSDU}_{j, h}=\frac{\sum_{i=1}^{k} n_{i} \times \frac{o_{i, j, h}}{e_{i, j, h}}}{\sum_{i=1}^{k} n_{i} \times \frac{1}{e_{i, j, h}}}
$$


The results of the RSDU are as follows:

- An RSDU of 1 indicates that only the dinucleotide of interest is being used in the sequence, all the other synonymous dinucleotides being absent for the given position;

- $\quad$ an RSDU of 0 , similar to the SDU, indicates that the dinucleotide of interest is completely absent in the given frame position.

\subsection{Measuring the Null Distribution}

The variance of the metric is expected to vary depending on the sequence length and amino acid composition. Thus, we have developed a method of measuring the null distribution by randomly populating the given sequence with synonymous codons without changing the amino acid sequence. This process takes place for a number of iterations, calculating the SDU (or RSDU) for each model sequence. This produces a distribution that represents the random error of the metric for the given sequence abiding to the null hypothesis.

\subsection{DinuQ Python Package}

We have developed a Python3 package called DinuQ (dinucleotide quantification) that includes modules for calculating the SDU and RSDU with the respective error distribution for any set of coding sequences provided in a FASTA file. The package further includes modules for calculating the RSCU [20] and relative dinucleotide abundance (RDA, sometimes called DRA) [23], so that the user can make comparisons between metrics. The calculated values can be provided in a Python dictionary to manipulate within the Python framework, or simply exported in a table format. The Biopython package [24] is used by the tool to facilitate sequence input. The package can be installed through the Python Package Index (PyPI). Full documentation and code are available online at https://github.com/ spyros-lytras/dinuq.

\subsection{Applying the SDU on Viral Sequences}

To test the uses of the SDU we have collected polyprotein coding sequences of vertebrate- and invertebrate-specific viruses of the Flaviviridae family, analysed in previous research [25]. Our analysis focuses on two representatives of the dataset: The insect-specific Aedes flavivirus (AEFV, Genbank accession: AB488408.1) that usually affects Aedes spp. mosquitoes [26], and the Apoi virus (APOIV, Genbank accession: AF160193.1) that has no known insect vector and affects rodents of the Apodemus genus [27]. To extend the analysis, we assembled a second dataset by collecting the coding sequences of all members of the Rhabdoviridae family included in the ICTV Virus Metadata Resource (version November 27, 2019; MSL34) [28], being labelled as having a vertebrate or invertebrate host. The SDU, RSDU, RDA and RSCU values were calculated using the DinuQ Python package. General Linear Models (GLM) for statistical comparisons were performed using the R coding language.

\section{Results}

\subsection{Effect of Sequence Length on SDU Error}

Because the SDU splits up the sequence information into many categories (i.e., independent proportions of the dinucleotide of interest for each amino acid or amino acid pair), if the given sequence is short there will be less information in each category and subsequently more variability/error in the calculated SDU value. We have randomly simulated 10 amino acid sequences of different lengths sequentially increasing by $10 \%$ of the longest sequence's length (i.e., from 700 to 7000 amino acids long). We then implemented our method of measuring error and calculated the SDU for each random sample. Figure 1a,b illustrate how the error distributions and their standard deviations vary between sequences of different lengths. By principle, the standard deviation of the error distribution should approach 0 as 
the sequence length increases. Our simulation experiment, however, shows that the magnitude of error is consistently very low for sequences longer than about 17,000 bp (standard deviation < 0.05 ).

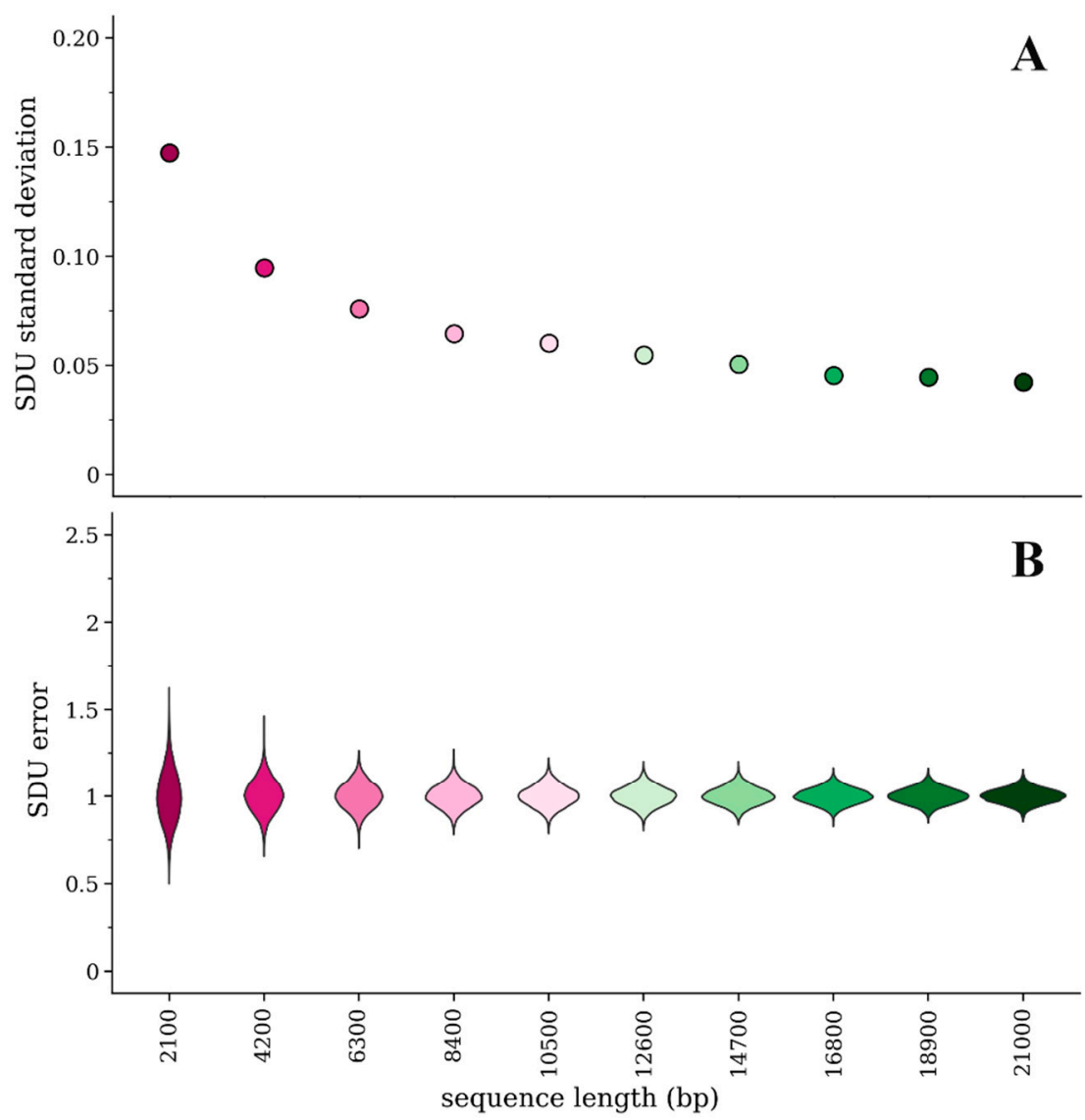

Figure 1. Comparison of error for the $\mathrm{SDU}_{\mathrm{CpGbridge}}$ of 10 simulated amino acid sequences of different lengths with 1000 random samples of nucleotide sequences for each amino acid sequence: (A) Standard deviation of the mean of the SDU error distributions; (B) Violin plots of the error distribution for each simulated sequence.

\subsection{Metric Comparisons Using an Insect- and a Vertebrate-Specific Flavivirus}

Previous analysis [25] showed that the host environment differently affects the nucleotide, codon and dinucleotide composition in viruses of the Flaviviridae family. In particular, APOIV and other flaviviruses with no known insect vector show an under-representation of $\mathrm{CpG}$, possibly a result of the ZAP-related vertebrate immune response targeting CpG-rich viruses. In contrast, AEFV and other insect-specific flaviviruses do not exhibit this bias. In Figures $2 b$ and $3 b$, we present the SDU patterns for all dinucleotides and frame positions of APOIV and AEFV, respectively. The SDU of all three frame positions of $\mathrm{CpG}$ in AEFV (Figure $3 b$ ) fall within the error distribution $\left(\mathrm{SDU}_{\mathrm{CpGbridge}}=0.96\right.$, error $_{\mathrm{MIN}}=0.85$, error $_{\mathrm{MAX}}=1.22 ; \mathrm{SDU}_{\mathrm{CpGpos} 1}=1.08$, error $_{\mathrm{MIN}}=0.84$, error $_{\mathrm{MAX}}=1.15 ; \mathrm{SDU}_{\mathrm{CpGpos} 2}$ $=0.87$, error $_{\mathrm{MIN}}=0.80$, error $\mathrm{MAX}=1.22$ ), whereas the respective SDU values in APOIV, the rodent infecting virus, (Figure $2 b)$ are well below $1\left(\mathrm{SDU}_{\mathrm{CpGbridge}}=0.41\right.$, error $\mathrm{MIN}=0.82$, error $_{\mathrm{MAX}}=1.19$; $\mathrm{SDU}_{\mathrm{CpGpos} 1}=0.56$, error $_{\mathrm{MIN}}=0.82$, error $_{\mathrm{MAX}}=1.19 ; \mathrm{SDU}_{\mathrm{CpGpos} 2}=0.30$, error $_{\mathrm{MIN}}=0.79$, error $_{\mathrm{MAX}}=$ 1.21), indicating significant $\mathrm{CpG}$ under-representation. These observations are in agreement with the previous research and the hypothesis of a CpG-targeting antiviral mechanism in vertebrates. 

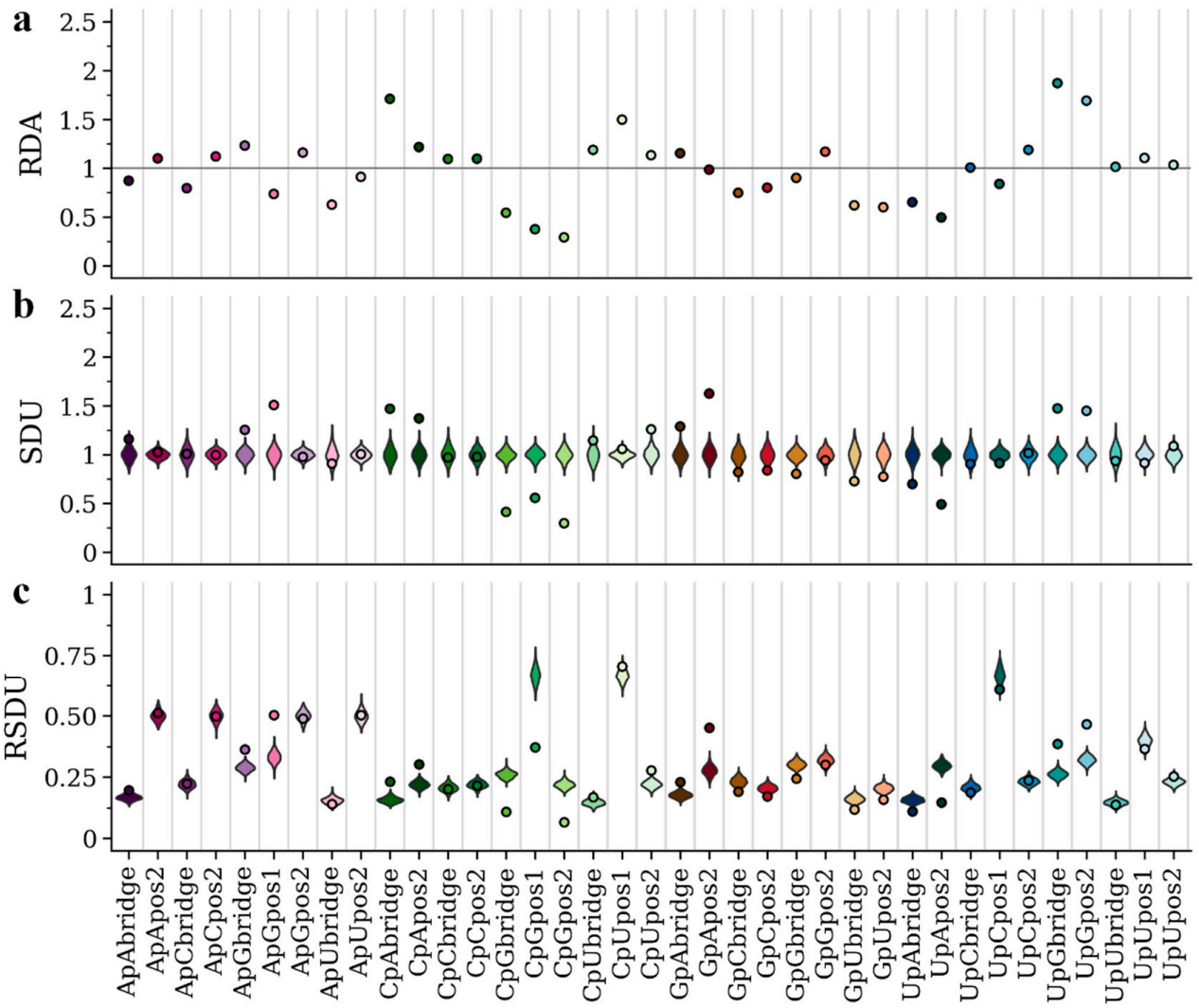

Figure 2. RDA (a), SDU (b) and RSDU (c) values for all informative dinucleotides and frame positions plotted for the APOIV coding sequence. Dot points indicate observed values and violin plots indicate SDU/RSDU error distributions around the null hypothesis (1000 random samples for each value). The grey horizontal line indicates an RDA of 1 . Position 1 of dinucleotides CpC, CpA, GpC, GpG, $\mathrm{GpU}, \mathrm{GpA}, \mathrm{UpG}, \mathrm{UpA}, \mathrm{ApC}, \mathrm{ApU}, \mathrm{ApA}$ are excluded because they can only produce one amino acid (non-informative).

We also present the RDA and RSDU values for the two viruses, in order to make comparisons between the metrics (Figure 2a,c and Figure 3a,c). In contrast to the SDU, RDA values cannot be compared to a null distribution, so there is no statistical evaluation of over- and under-representation of dinucleotides. For example, in Figure 2 the $\mathrm{CpU}$ dinucleotide seems to be over-represented in all three frame positions according to the RDA metric. The SDU plot, however, shows that CpU bridge and position 1 values fall within the null distributions and only position 2 can be confidently described as over-represented $\left(\mathrm{SDU}_{\mathrm{CpUpos} 2}=1.26\right)$. Thus, by using the SDU one can assess how confidently a value reflects over- or under-representation. For example, in Figure 3a, RDA suggests under-representation of position 2 and bridge of GpU and $\mathrm{UpA}\left(\mathrm{RDA}_{\mathrm{GpUbridge}}=0.86, \mathrm{RDA}_{\mathrm{GpUpos} 2}=0.76, \mathrm{RDA}_{\mathrm{UpAbridge}}=\right.$ $0.86, \mathrm{RDA}_{\mathrm{UpApos} 2}=0.71$ ). According to our metric, the $\mathrm{GpU}$ values fall within the null distribution $\left(\mathrm{SDU}_{\mathrm{GpUbridge}}=1.02\right.$, error $_{\mathrm{MIN}}=0.72$, error $_{\mathrm{MAX}}=1.28 ; \mathrm{SDU}_{\mathrm{GpUpos} 2}=0.96$, error $_{\mathrm{MIN}}=0.78$, error $_{\mathrm{MAX}}=$ 1.22 ) and only UpA can be confidently called under-represented in the AEFV genome (SDU UpAbridge $=$ 0.75, error $_{\mathrm{MIN}}=0.81$, error $_{\mathrm{MAX}}=1.24 ; \mathrm{SDU}_{\mathrm{UpApos} 2}=0.67$, error $_{\mathrm{MIN}}=0.84$, error $\left._{\mathrm{MAX}}=1.19\right)$ (Figure $\left.3 \mathrm{~b}\right)$. 

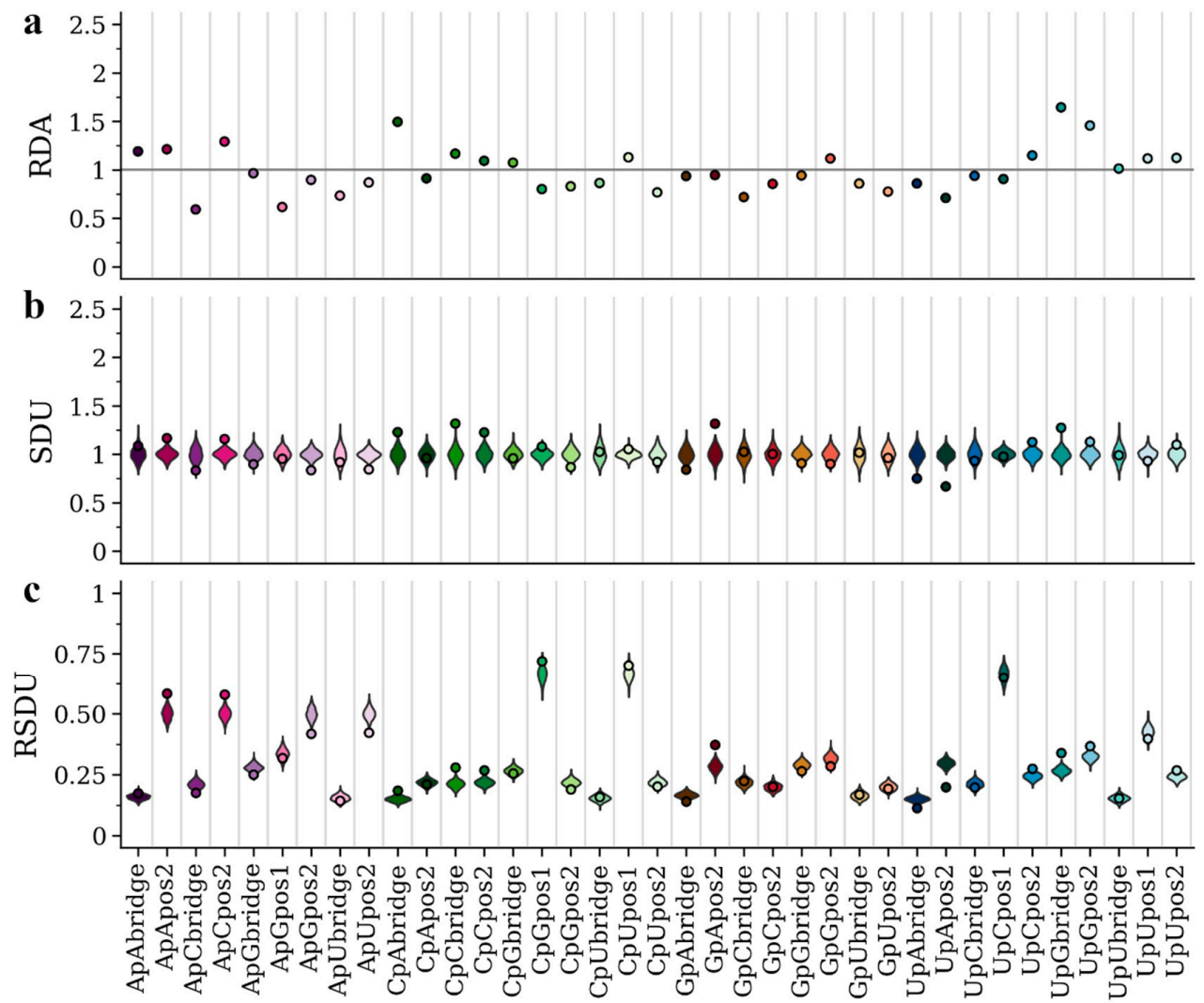

Figure 3. RDA (a), SDU (b) and RSDU (c) values for all informative dinucleotides and frame positions plotted for the AEFV coding sequence. Dot points indicate observed values and violin plots indicate SDU/RSDU error distributions around the null hypothesis (1000 random samples for each value). The grey horizontal line indicates an RDA of 1 . Position 1 of dinucleotides CpC, CpA, GpC, GpG, $\mathrm{GpU}, \mathrm{GpA}, \mathrm{UpG}, \mathrm{UpA}, \mathrm{ApC}, \mathrm{ApU}, \mathrm{ApA}$ are excluded because they can only produce one amino acid (non-informative).

The RSDU plots (Figures 2c and 3c) showcase how the relative expected number of occurrences differs between dinucleotides and frame positions. For example, for both viruses UpC representation falls within the null distribution for all three frame positions, however, the expected RSDU value under the null hypothesis is much larger for UpC position 1 (Figures $2 \mathrm{c}$ and $3 \mathrm{c}$ ). This simply depends on the expected occurrences of codons (or codon pairs for bridge position) that contain this dinucleotide at that frame position under synonymous codon usage. The RSDU can be useful when comparing the level of over-representation between two dinucleotide combinations. The dinucleotides UpG and CpA are both over-represented at the bridge position in the APOIV genome, with identical SDU values $\left(\mathrm{SDU}_{\mathrm{UpGbridge}}=1.47, \mathrm{SDU}_{\mathrm{CpAbridge}}=1.47\right)$. However, $\mathrm{UpG}$ is relatively more over-represented than $\mathrm{CPA}$, as indicated by their RSDU values $\left(\mathrm{RSDU}_{\mathrm{UpGbridge}}=0.38, \mathrm{RSDU}_{\mathrm{CpAbridge}}=0.23\right)$. The reason why the SDU values of the two are the same is because the maximum value $\mathrm{SDU}_{\mathrm{UpGbridge}}$ can take (when all synonymous bridge positions have a $\mathrm{UpG}$ ) is 3.33 , much lower than the $\mathrm{SDU}_{\mathrm{CpAbridge}}$ maximum value of 5.24 .

The SDU of a given dinucleotide should directly reflect the RSCU of the codons that contain it (or in case of the bridge position: the first nucleotide in the third codon position and second nucleotide in the first codon position of the downstream amino acid). To illustrate this relation between the two metrics, we have calculated the RSCU for all codons of AEFV and APOIV (Table 2). ApG in position 1 seems to be over-represented in APOIV (SDU ApGpos1 $=1.51$ ), which is not the case in AEFV (SDU ApGpos1 $_{\text {A }} 0.95$ ). This is clearly depicted in the RSCU values of all AG-starting codons being higher in APOIV (Table 2). AGA is highly over-represented in the APOIV genome exclusively (APOIV: RSCU $=2.19$; AEFV: RSCU $=1.00$ ), which also explains the high SDU value for GpA in frame position 2 (APOIV: SDU GpApos2 
= 1.63; AEFV: $\mathrm{SDU}_{\mathrm{GpApos} 2}=0.96$ ). This over-representation of AG-starting codons in APOIV also seems to drive the only clear inconsistency between SDU and RDA values, where position $1 \mathrm{ApG}$ is under-represented based on RDA but over-represented based on SDU (Figure 2a,b). All CG-starting and -ending codons are under-represented in APOIV (Table 2), consistent with CpG depletion and low $\mathrm{SDU}_{\mathrm{CpGpos} 1}$ and $\mathrm{SDU} \mathrm{CpGpos} 2_{\mathrm{in}}$ the vertebrate-infecting virus. While it is seemingly easy to trace such dinucleotide bias patterns in the 1st and 2nd frame position only by using the RSCU metric, this becomes much harder for the bridge position. For example, the SDU values for the bridge positions of ApG, GpA and CpG also fall outside the null hypothesis distribution in APOIV, but not in AEFV (Figures $2 \mathrm{~b}$ and $\mathrm{3b}$ ). This highlights the importance of having a metric for detecting compositional biases on the dinucleotide level.

Table 2. RSCU values for each codon, calculated for the APOIV and AEFV coding sequences. Highlighted in bold are the values mentioned in the text. No values have been calculated for stop codons (UAA, UAG, UGA), since only one coding sequence was used for each virus.

\begin{tabular}{lcclcccccccc}
\hline & APOIV & AEFV & & APOIV & AEFV & & APOIV & AEFV & & APOIV & AEFV \\
\hline UUU & 1.11 & 1.08 & UCU & 1.01 & 0.75 & UAU & 0.98 & 1.00 & UGU & 0.95 & 1.04 \\
UUC & 0.89 & 0.92 & UCC & 0.73 & 1.13 & UAC & 1.02 & 1.00 & UGC & 1.05 & 0.96 \\
UUA & 0.38 & 0.56 & UCA & 1.57 & 0.96 & UAA & STOP & STOP & UGA & STOP & STOP \\
UUG & 1.39 & 1.23 & UCG & $\mathbf{0 . 3 4}$ & $\mathbf{1 . 0 6}$ & UAG & STOP & STOP & UGG & 1.00 & 1.00 \\
CUU & 1.08 & 0.83 & CCU & 1.19 & 0.89 & CAU & 1.25 & 1.05 & CGU & $\mathbf{0 . 4 8}$ & $\mathbf{1 . 1 9}$ \\
CUC & 1.00 & 1.42 & CCC & 0.86 & 1.00 & CAC & 0.75 & 0.95 & CGC & $\mathbf{0 . 3 9}$ & $\mathbf{1 . 1 9}$ \\
CUA & 0.67 & 0.79 & CCA & 1.69 & 1.32 & CAA & 0.91 & 1.23 & CGA & $\mathbf{0 . 6 8}$ & $\mathbf{1 . 0 5}$ \\
CUG & 1.48 & 1.17 & CCG & $\mathbf{0 . 2 5}$ & $\mathbf{0 . 7 9}$ & CAG & 1.09 & 0.77 & CGG & $\mathbf{0 . 6 8}$ & $\mathbf{0 . 8 8}$ \\
AUU & 1.10 & 1.22 & ACU & 1.13 & 0.98 & AAU & 0.91 & 0.84 & AGU & $\mathbf{1 . 0 4}$ & $\mathbf{0 . 9 6}$ \\
AUC & 1.15 & 1.05 & ACC & 1.27 & 1.07 & AAC & 1.09 & 1.16 & AGC & $\mathbf{1 . 3 2}$ & $\mathbf{1 . 1 3}$ \\
AUA & 0.75 & 0.73 & ACA & 1.29 & 1.00 & AAA & 1.01 & 1.23 & AGA & $\mathbf{2 . 1 9}$ & $\mathbf{1 . 0 0}$ \\
AUG & 1.00 & 1.00 & ACG & $\mathbf{0 . 3 2}$ & $\mathbf{0 . 9 5}$ & AAG & 0.99 & 0.77 & AGG & $\mathbf{1 . 5 8}$ & $\mathbf{0 . 6 9}$ \\
GUU & 1.08 & 1.29 & GCU & 1.65 & 1.07 & GAU & 0.97 & 0.67 & GGU & 0.73 & 0.74 \\
GUC & 1.00 & 0.98 & GCC & 1.03 & 1.62 & GAC & 1.03 & 1.33 & GGC & 0.73 & 0.73 \\
GUA & 0.29 & 0.62 & GCA & 1.05 & 0.68 & GAA & 1.09 & 1.07 & GGA & 1.75 & 1.57 \\
GUG & 1.63 & 1.11 & GCG & $\mathbf{0 . 2 7}$ & $\mathbf{0 . 6 3}$ & GAG & 0.91 & 0.93 & GGG & 0.80 & 0.96 \\
\hline
\end{tabular}

\subsection{SDU Shows Consistent CpG Differences between Insect- and Vertebrate-Specific Viruses}

Since there is evidence for a vertebrate immune response selecting against $\mathrm{CpG}$ dinucleotides in viral genomes, we decided to further explore this trend between members of the Flaviviridae family, specific to and absent in vertebrate hosts using the SDU. First, we calculated the SDU of CpG for all frame positions for the two sets of insect-specific and vertebrate-specific (no known insect vector) viruses used by Simón et al. [25]. Figure 4a shows the SDU values for the two groups plotted against the total GC content of the viral coding sequences. As indicated by the graph, the CpG SDU correlates with the overall GC content with slopes that are not significantly different between hosts (GLM: $F_{1,57}=1.492$, $p=0.227$ ). However, the intercepts of the fitted lines are significantly different between the host groups with the host and the GC content predictors explaining $89 \%$ of the variance. The vertebrate-infecting viruses have significantly lower SDU values, thus more $\mathrm{CpG}$ depletion than invertebrate-specific flaviviruses (GLM: $R^{2}=0.89, \mathrm{~F}_{2,57}=238.3, p<0.0001$ ). 


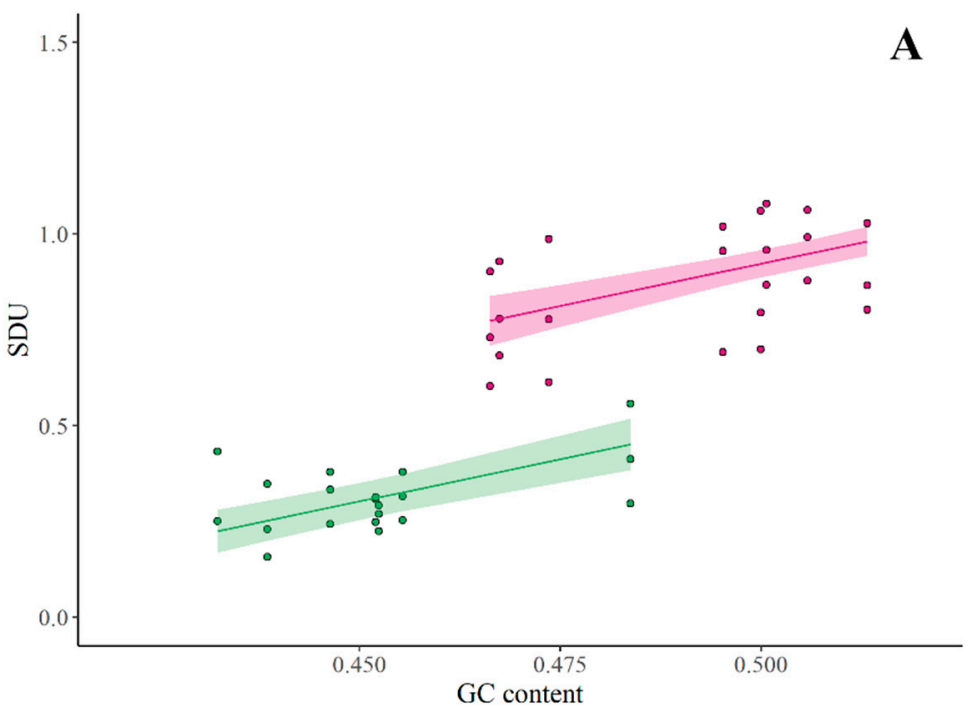

Host

Invertebrate

Vertebrate

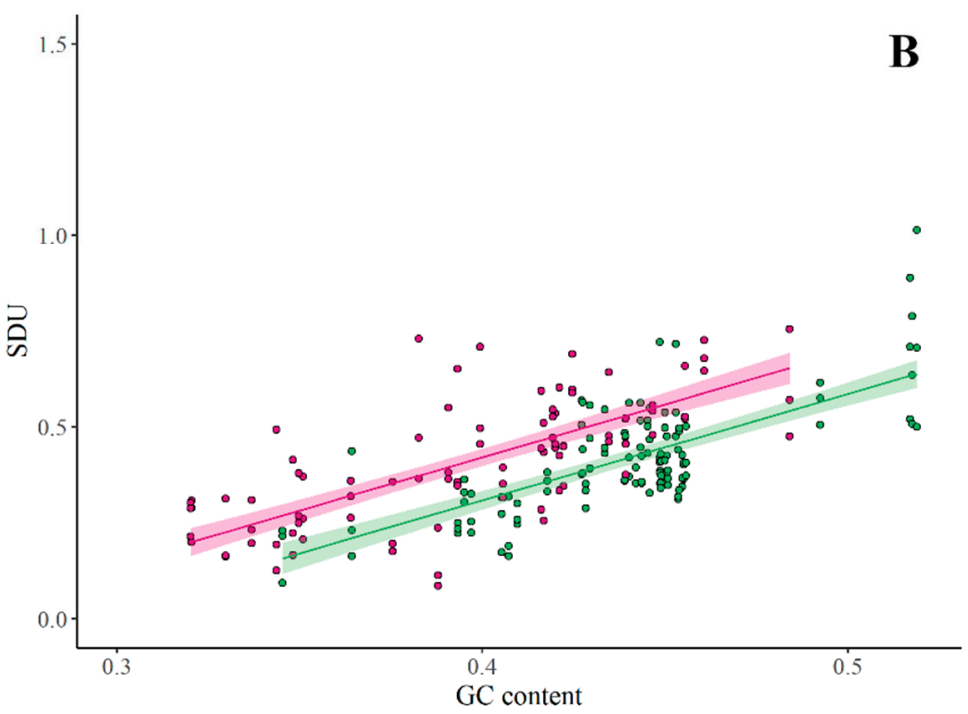

Figure 4. Comparison of $\mathrm{SDU}_{\mathrm{CpG}}$ for all frame positions between invertebrate- and vertebrate-specific (A) Flaviviridae and (B) Rhabdoviridae, plotted against the overall GC content of the coding sequences (Supplementary Table S1).

This analysis was replicated on a set of viruses of the Rhabdoviridae family (Figure $4 \mathrm{~b}$ ). The trend observed is similar to that seen with the Flaviviridae. Both GC content and host-specificity explain $51 \%$ of the variance (GLM: $R^{2}=0.51, \mathrm{~F}_{2,201}=105.4, p<0.0001$ ) around the CpG representation as quantified by our metric. The difference in rhabdovirus $\mathrm{CpG}$ depletion is not as large as in flaviviruses. $\mathrm{CPG}$ representation in invertebrate-infecting Flaviviridae is consistent with the null hypothesis of equal synonymous dinucleotides (mean $\mathrm{SDU}_{\mathrm{CpG}}=0.94$ ), while vertebrate-infecting members of the family show a higher $\mathrm{CpG}$ suppression (mean $\mathrm{SDU}_{\mathrm{CpG}}=0.31$ ). On the contrary, both sets of Rhabdoviridae have depleted $\mathrm{CpG}$ levels, vertebrate-specific ones having slightly more suppression (invertebrate-specific: mean $\mathrm{SDU}_{\mathrm{CpG}}=0.42$, vertebrate-specific: mean $\left.\mathrm{SDU}_{\mathrm{CpG}}=0.41\right)$.

\section{Discussion}

In this paper we propose SDU as a novel method for quantifying dinucleotide representation in a coding sequence by comparing the observed frequency of a synonymous dinucleotide to that expected under the null hypothesis of equal synonymous codon usage. We further extend this metric 
for comparisons between different dinucleotides with the RSDU formula and provide a means of measuring statistical error for the calculated values.

Using the coding sequences of two flaviviruses with different dinucleotide compositions, APOIV and AEFV, we present how the SDU compares to its extension, RSDU, and to the standard metric for dinucleotide quantification, RDA (Figures 2 and 3). The RSDU can prove useful for comparing the level of over-representation between two dinucleotides, bypassing the variable scale of SDU values for different dinucleotide positions. Overall, there is agreement between the results of RDA and SDU. RDA values close to 1 , however, can be misinterpreted as over- or under-representation when the value is not exactly equal to 1 just by chance. Our method provides a way of testing the significance of a value by comparing to a null distribution and accepting a skew in dinucleotide representation if the SDU value falls outside the expected distribution. This might be a more conservative way of assessing dinucleotide representation but avoids such misinterpretations.

We have explored the representation of different dinucleotides in a set of previously examined members of the Flaviviridae family, using the SDU, and produced results in agreement with previous research [25]. Based on suggestions from current research that a host-mediated antiviral response in vertebrates selects against CpGs in viral RNA, we focused on examining CpG representation in the Flaviviridae and a set of Rhabdoviridae viruses. In both datasets, the SDU calculations show a greater depletion of $\mathrm{CpG}$ in the vertebrate-specific viruses. Even though there is no experimental evidence that a CpG-recognising immune response is acting on flaviviruses or rhabdoviruses in vertebrates, other RNA viruses seem to be affected by such a mechanism $[10,12,29]$. Thus, we hypothesise that this immune mechanism is responsible for the difference in CPG SDU values between the two virus groups.

It is important to consider that factors other than a dinucleotide-targeting immune response are likely to bias viral genomic composition and such biases might also be reflected in the SDU values. For example, most invertebrate flaviviruses show weak or no CpG under-representation, while vertebrate-infecting flaviviruses show high $\mathrm{CpG}$ depletion. This pattern is less clear in the rhabdoviruses, where $\mathrm{CpG}$ is under-represented in both groups. We suggest this might be a result of other rhabdovirus-specific host mechanisms, or simply a lineage-specific mutational bias or the base composition of the Rhabdoviridae.

Finally, with the SDU, each informative frame position is examined separately for a given dinucleotide. This feature should be useful for exploring cases where selective or mutational processes are acting on specific positions in the coding sequence, or on longer motifs including the dinucleotide of interest. In comparison to the RDA, the SDU takes into account where changes due to a dinucleotide preference are more likely to take place, by assuming that synonymous nucleotide changes are more likely than non-synonymous ones. Thus, our metric reflects dinucleotide patterns, while being informed by a biological assumption. Interpretations of the metric should still be made with caution, since it does not disentangle between the forces affecting synonymous dinucleotide representation, whether that is selection or mutation on the dinucleotide level, or forces targeting codons and longer motifs. Whilst we have developed this metric to expand our understanding of dinucleotide biases in viruses, the SDU can have broader applications, for example in the host coding sequences.

Supplementary Materials: The following are available online at http://www.mdpi.com/1999-4915/12/4/462/s1, Table S1: virus names, NCBI accessions and SDU values for Figure 4.

Author Contributions: Conceptualization, S.L. and J.H.; methodology, S.L.; software, S.L.; validation, S.L.; formal analysis, S.L. and J.H.; writing-original draft preparation, S.L.; writing — review and editing, J.H.; visualization, S.L.; supervision, J.H.; project administration, J.H. All authors have read and agreed to the published version of the manuscript.

Funding: This research was funded by the Medical Research Council (MC_UU_12014/12).

Acknowledgments: We thank Richard Reeve, Paul Johnson and Richard Orton for feedback on the approach and Oscar MacLean for providing feedback on the manuscript.

Conflicts of Interest: The authors declare no conflict of interest. The funders had no role in the design of the study; in the collection, analyses, or interpretation of data; in the writing of the manuscript, or in the decision to publish the results. 
Computer Code and Software: The DinuQ Python package is available to install through PyPI, under the name "dinuq" (https://pypi.org/project/dinuq/). All code is available on GitHub (https://github.com/spyros-lytras/dinuq).

\section{References}

1. Beutler, E.; Gelbart, T.; Han, J.H.; Koziol, J.A.; Beutler, B. Evolution of the genome and the genetic code: Selection at the dinucleotide level by methylation and polyribonucleotide cleavage. Proc. Natl. Acad. Sci. USA 1989, 86, 192-196. [CrossRef]

2. Karlin, S.; Doerfler, W.; Cardon, L.R. Why is CpG suppressed in the genomes of virtually all small eukaryotic viruses but not in those of large eukaryotic viruses? J. Virol. 1994, 68, 2889-2897. [CrossRef] [PubMed]

3. Cheng, X.; Virk, N.; Chen, W.; Ji, S.; Ji, S.; Sun, Y.; Wu, X. CpG Usage in RNA Viruses: Data and Hypotheses. PLOS ONE 2013, 8, e74109. [CrossRef] [PubMed]

4. Bird, A. DNA methylation and the frequency of CpG in animal DNA. Nucleic Acids Res. 1980, 8, 1499-1504. [CrossRef] [PubMed]

5. Cooper, D.N.; Krawczak, M. Cytosine methylation and the fate of CpG dinucleotides in vertebrate genomes. Qual. Life Res. 1989, 83, 181-188. [CrossRef]

6. Shaw, G.; Kamen, R. A conserved AU sequence from the 3' untranslated region of GM-CSF mRNA mediates selective mRNA degradation. Cell 1986, 46, 659-667. [CrossRef]

7. Duan, J.; Antezana, M. Mammalian Mutation Pressure, Synonymous Codon Choice, and mRNA Degradation. J. Mol. Evol. 2003, 57, 694-701. [CrossRef]

8. Simmonds, P.; Xia, W.; Baillie, J.K.; McKinnon, K. Modelling mutational and selection pressures on dinucleotides in eukaryotic phyla -selection against CpG and UpA in cytoplasmically expressed RNA and in RNA viruses. BMC Genom. 2013, 14, 610. [CrossRef]

9. Atkinson, N.J.; Witteveldt, J.; Evans, D.J.; Simmonds, P. The influence of CpG and UpA dinucleotide frequencies on RNA virus replication and characterization of the innate cellular pathways underlying virus attenuation and enhanced replication. Nucleic Acids Res. 2014, 42, 4527-4545. [CrossRef]

10. Tulloch, F.; Atkinson, N.J.; Evans, D.J.; Ryan, M.D.; Simmonds, P. RNA virus attenuation by codon pair deoptimisation is an artefact of increases in CpG/UpA dinucleotide frequencies. eLife 2014, 3, 04531. [CrossRef]

11. Witteveldt, J.; Martin-Gans, M.; Simmonds, P. Enhancement of the Replication of Hepatitis C Virus Replicons of Genotypes 1 to 4 by Manipulation of CpG and UpA Dinucleotide Frequencies and Use of Cell Lines Expressing SECL14L2 for Antiviral Resistance Testing. Antimicrob. Agents Chemother. 2016, 60, 2981-2992. [CrossRef]

12. Gaunt, E.; Wise, H.M.; Zhang, H.; Ni Lee, L.; Atkinson, N.J.; Nicol, M.Q.; Highton, A.J.; Klenerman, P.; Beard, P.; Dutia, B.M.; et al. Elevation of $\mathrm{CpG}$ frequencies in influenza A genome attenuates pathogenicity but enhances host response to infection. eLife 2016, 5. [CrossRef]

13. Klitting, R.; Riziki, T.; Moureau, G.; Piorkowski, G.; A Gould, E.; De Lamballerie, X. Exploratory re-encoding of yellow fever virus genome: New insights for the design of live-attenuated viruses. Virus Evol. 2018, 4, vey021. [CrossRef] [PubMed]

14. Takata, M.A.; Carneiro, D.G.; Zang, T.M.; Soll, S.J.; York, A.; Blanco-Melo, D.; Bieniasz, P.D. CG dinucleotide suppression enables antiviral defence targeting non-self RNA. Nature 2017, 550, 124-127. [CrossRef] [PubMed]

15. Odon, V.; Fros, J.J.; Goonawardane, N.; Dietrich, I.; Ibrahim, A.; Alshaikhahmed, K.; Nguyen, D.; Simmonds, P. The role of ZAP and OAS3/RNAseL pathways in the attenuation of an RNA virus with elevated frequencies of CPG and UpA dinucleotides. Nucleic Acids Res. 2019, 47, 8061-8083. [CrossRef] [PubMed]

16. Lin, Y.-T.; Chiweshe, S.; McCormick, D.; Raper, A.R.; Wickenhagen, A.; DeFillipis, V.; Gaunt, E.; Simmonds, P.; Wilson, S.J.; Grey, F.; et al. Human cytomegalovirus evades ZAP detection by suppressing CpG dinucleotides in the major immediate early genes. bioRxiv 2020. [CrossRef]

17. Shackelton, L.A.; Parrish, C.R.; Holmes, E.C. Evolutionary Basis of Codon Usage and Nucleotide Composition Bias in Vertebrate DNA Viruses. J. Mol. Evol. 2006, 62, 551-563. [CrossRef]

18. Fros, J.; Dietrich, I.; Alshaikhahmed, K.; Passchier, T.; Evans, D.J.; Simmonds, P. CpG and UpA dinucleotides in both coding and non-coding regions of echovirus 7 inhibit replication initiation post-entry. eLife 2017, 6 . [CrossRef] 
19. Sharp, P.M.; Averof, M.; Lloyd, A.T.; Matassi, G.; Peden, J.F. DNA sequence evolution: The sounds of silence. Philos. Trans. R. Soc. B Boil. Sci. 1995, 349, 241-247. [CrossRef]

20. Sharp, P.M.; Tuohy, T.M.; Mosurski, K.R. Codon usage in yeast: Cluster analysis clearly differentiates highly and lowly expressed genes. Nucleic Acids Res. 1986, 14, 5125-5143. [CrossRef]

21. Greenbaum, B.D.; Levine, A.J.; Bhanot, G.; Rabadan, R. Patterns of Evolution and Host Gene Mimicry in Influenza and Other RNA Viruses. PLOS Pathog. 2008, 4, e1000079. [CrossRef] [PubMed]

22. Greenbaum, B.D.; Cocco, S.; Levine, A.; Monasson, R. Quantitative theory of entropic forces acting on constrained nucleotide sequences applied to viruses. Proc. Natl. Acad. Sci. USA 2014, 111, 5054-5059. [CrossRef] [PubMed]

23. Kariin, S.; Burge, C. Dinucleotide relative abundance extremes: A genomic signature. Trends Genet. 1995, 11, 283-290. [CrossRef]

24. Cock, P.J.; Antao, T.; Chang, J.T.; Chapman, B.; Cox, C.J.; Dalke, A.; Friedberg, I.; Hamelryck, T.; Kauff, F.; Wilczynski, B.; et al. Biopython: Freely available Python tools for computational molecular biology and bioinformatics. Bioinformatics 2009, 25, 1422-1423. [CrossRef]

25. Simón, D.; Fajardo, Á.; Soñora, M.; Delfraro, A.; Musto, H. Host influence in the genomic composition of flaviviruses: A multivariate approach. Biochem. Biophys. Res. Commun. 2017, 492, 572-578. [CrossRef]

26. Blitvich, B.; Firth, A.E. Insect-Specific Flaviviruses: A Systematic Review of Their Discovery, Host Range, Mode of Transmission, Superinfection Exclusion Potential and Genomic Organization. Viruses 2015, 7, 1927-1959. [CrossRef]

27. Billoir, F.; De Micco, P.; Tolou, H.; De Chesse, R.; De Lamballerie, X.; Gould, E.A. Phylogeny of the genus Flavivirus using complete coding sequences of arthropod-borne viruses and viruses with no known vector. J. Gen. Virol. 2000, 81, 781-790. [CrossRef]

28. International Committee on Taxonomy of Viruses (ICTV). Available online: https://talk.ictvonline.org/ taxonomy/vmr/ (accessed on 27 February 2020).

29. Ibrahim, A.; Fros, J.; Bertran, A.; Sechan, F.; Odon, V.; Torrance, L.; Kormelink, R.; Simmonds, P. A functional investigation of the suppression of $\mathrm{CpG}$ and $\mathrm{UpA}$ dinucleotide frequencies in plant RNA virus genomes. Sci. Rep. 2019, 9, 1-14. [CrossRef]

(C) 2020 by the authors. Licensee MDPI, Basel, Switzerland. This article is an open access article distributed under the terms and conditions of the Creative Commons Attribution (CC BY) license (http://creativecommons.org/licenses/by/4.0/). 